\title{
Evaluation of superoxide dismutase 3, heme-oxygenase, and myeloperoxidase expression levels associated with oxidative stress in chronic periodontitis
}

\author{
Yong Yoon ${ }^{1}$, Hyerin $\mathrm{Kim}^{2}$, and Jae Mok Lee ${ }^{3 *}$ \\ ${ }^{1}$ Master's Degree, Department of Periodontology, School of Dentistry, Kyungpook National University, Daegu, Republic of Korea \\ ${ }^{2}$ Resident, Department of Periodontology, School of Dentistry, Kyungpook National University, Daegu, Republic of Korea \\ ${ }^{3}$ Professor, Department of Periodontology, School of Dentistry, Kyungpook National University, Daegu, Republic of Korea
}

This study aimed to investigate the expression levels of antioxidative enzymes such as superoxide dismutase 3 (SOD3), heme-oxygenase (HO), and myeloperoxidase (MPOs), which are known to be associated with oxidative stress induction in chronic inflammatory conditions like periodontitis. We compared the expression levels of SOD3, HO, and MPO in the periodontal tissues of healthy individuals with those in the periodontal tissues of chronic periodontitis patients. The expression levels of SOD3, HO, and MPO were determined by Western blot technique, and the data were statistically analyzed. The expression levels of SOD3 and HO increased in periodontitis patients compared with those in healthy controls, and the degree of increase was dependent on the severity of periodontitis $(p<0.05)$. In contrast, the expression level of MPO was lower in the periodontal tissues of healthy controls than that in the periodontal tissues of periodontitis patients $(p<0.05)$. SOD and $\mathrm{HO}$ are involved in oxidative stress via antioxidative activity, and MPO is involved in oxidative stress via peroxidative activity. Hence, these findings suggest that antioxidative activity depends on the severity of inflammation. In conclusion, SOD3, HO, and MPO may play a role in oxidative stress-induced periodontitis, and these three factors may be related to the severity of inflammation. These factors are expected to be applied to the diagnosis and treatment of periodontitis through more comprehensive studies.

Key Words: Chronic periodontitis; Heme oxygenase; Myeloperoxidase; Oxidative stress; Superoxide dismutase

(c) This is an open-access article distributed under the terms of the Creative Commons Attribution Non-Commercial License (http://creativecommons.org/licenses/by-nc/4.0) which permits unrestricted noncommercial use, distribution, and reproduction in any medium, provided the original work is properly cited.

\section{Introduction}

Periodontitis is a chronic inflammatory condition which accompanies loss of connective tissue attachment between the root and the alveolar bone [1,2]. The destruction of periodontal ligament tissue results from activitiy of various inflammatory factors and their interaction, and it is an im- portant part of the progression of periodontitis.

Superoxide dismutase (SOD) is an enzyme that alternately catalyzes the cleavage of superoxide radicals into oxygen $\left(\mathrm{O}_{2}\right)$ or hydrogen peroxide $\left(\mathrm{H}_{2} \mathrm{O}_{2}\right)$. Superoxide is produced as a by-product of oxygen metabolism and causes various kinds of cell damage. Hydrogen peroxide also causes cell damage and is degraded by other enzymes such as catalase.

Received March 4, 2021; Revised [1] April 25, 2021; [2] May 18, 2021; [3] June 7, 2021; Accepted June 10, 2021

*Corresponding author: Jae Mok Lee, Department of Periodontics, School of Dentistry, Kyungpook National University, 2175 Dalgubeol-daero, Jung-gu, Daegu 41940, Republic of Korea.

Tel: +82-53-600-7522, Fax: +82-53-427-3263, E-mail: leejmperio@gmail.com 
With this mechanism, SOD acts as an important antioxidant defense agent in most living cells exposed to oxygen [3]. Human leukocytes use enzymes such as NADPH oxidase to generate superoxide and other reactive oxygen species to kill bacteria. When the body is infected, some bacteria protect themselves by producing SOD. SOD exists in three different isoforms in human body. Among them, SOD3 is distributed in extracellular, unlike the others, but its definite role is ambiguous. Therefore, it is needed to investigate the association of SOD3 expression with increased expression under oxidative stress, since bone destruction patterns increase with the depth of periodontitis and adherence loss occurs $[4,5]$.

Heme-oxygenase $(\mathrm{HO})$ is enzyme which catalyzes the first-rate limiting step of degradation of hemoglobin pigment, heme [6]. It is an antioxidant such as the well-known SOD family and also known to be expressed in most living cells exposed to oxygen [7,8].

Myeloperoxidase (MPO) is a peroxidase enzyme that is transcribed by the MPO gene in humans. It is most abundantly expressed in neutrophil granules (subpopulations of leukocytes) and produces hypohalous acid ( $\mathrm{HOCl})$ for antimicrobial action. MPO is a member of the exportins (XPO) family, which produces $\mathrm{HOCl}$ as $\mathrm{H}_{2} \mathrm{O}_{2}$ and chloride anion (Cl-) during neutrophil expectoration and heme as a cofactor [9]. Deficiency of MPO is a genetic problem of enzymes that cause immune defects. In the medical field, it is used as a sensitive predictor of myocardial infarction and is used for the diagnosis of acute myeloid leukemia. Its expression mechanism is related with cofactor of SOD3 and HO mentioned above and antimicrobial function. Therefore, it can be expected to contribute to the development of medicines that can be used for periodontal diagnosis and periodontal treatment $[10,11]$.

Therefore, the roles of SOD3 and HO, and MPO, which are enzymes associated with oxidative stress on the development of periodontitis needs to be elucidated. In this study, we investigated the expression and interactions of SOD3, HO, and MPO in the healthy and various severity of inflammation tissue.

\section{Materials and Methods}

\section{Study population and tissue sampling}

Gingival specimens were obtained from patients with periodontal disease and from healthy individuals who visited Department of Periodontics, Kyungpook National University Dental Hospital. The exclusion criteria were specified as follows: pregnancy, smokers and any kind of systemic disease that could affect the response to treatment (diabetes mellitus, hypertension, cardiovascular disease, arthritis, liver disease, renal disease, malignant tumor, autoimmune disease and metabolic bone disease, etc.). Specimen were obtained during periodontal surgery including surgical crown lengthening or tooth extraction by internal bevel incision. The gingival samples were consisted of junctional and crevicular epithelium and connective tissue. All subjects agreed with informed consent before any surgical procedures.

The subjects were diagnosed and divided according to the severity of periodontitis based on the clinical and radiographic criteria. The clinical parameters like sulcus bleeding index (SBI) value [12] and probing pocket depth (PD) [13] were measured to determine clinical criteria. From the available radiographs, level of bone resorption was observed. Each of 16 subjects were included in 3 groups, and tissue were collected from the subjects. Classification followed the criteria of Mühlemann and Son [12] in 1971.

1) Normal (group I, control group, $n=16$ ): clinically healthy gingiva without bleeding (SBI 0,1) with no evidence of bone resorption, periodontal pockets or loss of attachment $(\mathrm{PD} \leq 3 \mathrm{~mm})$

2) Moderate chronic periodontitis (group II, $n=16$ ): chronic periodontitis showing evidence of loss of attachment and alveolar bone resorption, $3 \mathrm{~mm}<\mathrm{PD} \leq 6 \mathrm{~mm}$, and gingival inflammation (SBI 3)

3) Severe chronic periodontitis (group III, $n=16$ ): chronic periodontitis showing clear evidence of loss of attachment and alveolar bone resorption, PD> $6 \mathrm{~mm}$, and gingival inflammation (SBI 3)

This study was approved by the Ethical Committee of clinical experiments, Kyungpook national University Hospital (KNUH 2013-05-036-002). 
After periodontal surgery, tissue specimens were immediately placed on liquid nitrogen and subsequently frozen $\left(-70^{\circ} \mathrm{C}\right)$.

\section{Protein isolation and immunoblotting (Western blot analysis)}

For Western blotting, as previously described [14,15], frozen tissue samples were homogenized in RIPA lysis buffer (10 mM EDTA, 0.15 M NaCl) with 1:30 diluted protease inhibitor cocktail (Roche, Germany) [16]. The lysates were centrifuged at 12,000 $\mathrm{g}$ for 15 minutes at $4^{\circ} \mathrm{C}$. Bradford protein assay (Quick Start; BIO-RAD, Hercules, CA, USA) was performed for quantitative analysis of protein concentrations of supernatant liquid by a using Bovine serum albumin as standard.

Lysates were boiled for 3 minutes in sodium dodecyl sulfate (SDS) samples buffer (1 M Tris-Cl [pH 6.8], 40\% glycerol, 8\% SDS, 2\% mercapto-ethanol, 0.002\% Bromophenole blue). Prepared samples were divided by electrophoresis on 15\% SDS-polyacrylamide gels using gel, which maintains polypeptides in a denatured state, and were transferred to a polyvinylidene difluride membrane to make the proteins accessible to antibody detection.

The membranes was blocked in Tris-buffered saline (TBS) with a minute percentage of detergent such as Tween 20 containing 5\% powdered milk for 1 hour. Then, goat polyclonal anti-SOD3, anti-HO, and anti-MPO antibody (diluted 1:1,000 in TBS; Santa Cruz Biotechnology, Inc., Santa Cruz, CA, USA) were incubated with the membranes for overnight at $4^{\circ} \mathrm{C}$.

The membranes were rinsed in TBS with Tween 20 three times for 10 minutes and incubated with a horseradish peroxidase-linked donkey anti-goat secondary antibody (diluted 1: 2,000 in TBS) for 1 hour at room temperature. The membranes were washed three times for 10 minutes with TBS with Tween 20. The membranes were developed in an ECL Plus development kit (Amsterdam, Beckinghamshire, UK).

The quantitative analysis of SOD3, HO and MPO along with their $\beta$-actin (Abcam, Cambridge, UK) was performed using a densitometer (Image Gauge V 3.46, Koshin Graphic Systems; Fuji Photo Film Co., Tokyo, Japan). After normalization of each sample, comparative levels of SOD3, $\mathrm{HO}$, and MPO were expressed as a ratio of $\mathrm{SOD} 3, \mathrm{HO}$ and $\mathrm{MPO} /$ $\beta$-actin. Then the differences among groups were evaluated.

\section{Statistical analysis of the Western blot results}

All data were presented as means \pm standard deviation and statistically analyzed. One way ANOVA was used to compare the SOD3, HO, and MPO levels of 3 groups. $p^{-}$ value $<0.05$ was considered to be statistically significant. The correlations between gingival SOD3, HO, and MPO levels were evaluated and compared using Pearson's correlation coefficients. $p<0.05$ was considered significant.

\section{Results}

Demographic variable of the patients are shown in Table 1. The gingival tissue from the patients of normal group (group I), moderate periodontitis group (group II), and severe periodontitis group (group III) showed the expression of SOD3 corresponding to molecular weight about $52 \mathrm{kDa}$ in all samples. One representative band of SOD3 is shown Fig. 1. The expression levels of SOD3 were increased in or-

Table 1. Demographic characteristic of the study subjects

\begin{tabular}{lccc}
\hline Variable & $\begin{array}{c}\text { Group I Normal } \\
(\mathbf{n}=\mathbf{1 6})\end{array}$ & $\begin{array}{c}\text { Group II } \\
\text { Moderate periodontitis (n=16) }\end{array}$ & $\begin{array}{c}\text { Group III } \\
\text { Severe periodontitis (n=16) }\end{array}$ \\
\hline Sex, M/F & $10 / 6$ & $8 / 8$ & $7 / 9$ \\
Age (y) & $51.44 \pm 11.36^{\mathrm{a}}$ & $54.92 \pm 7.21^{\mathrm{b}}$ & $53.34 \pm 8.79^{\mathrm{c}}$ \\
Probing PD & $\mathrm{PD} \leq 3 \mathrm{~mm}$ & $3 \mathrm{~mm}<\mathrm{PD} \leq 6 \mathrm{~mm}$ & $\mathrm{PD}>6 \mathrm{~mm}$ \\
\hline
\end{tabular}

Values are presented as number only or mean \pm standard deviation.

M, male; F, female; PD, pocket depth.

${ }^{a}$ From 22 to 69 years. ${ }^{b}$ From 38 to 64 years. ${ }^{\text {cFom }} 35$ to 66 years. 


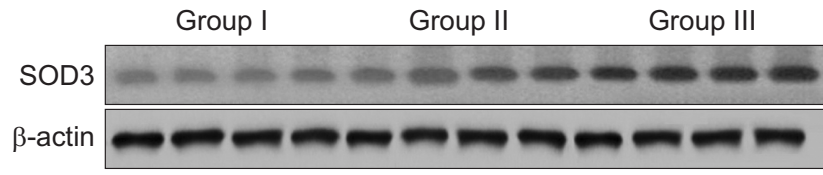

Fig. 1. Western analysis of superoxide dismutase 3 (SOD3) showing 4 representative samples in each group. SOD3 levels were quantified on the basis of $\beta$-actin levels. Group I: normal gingiva of healthy periodontal tissue. Group II: inflamed gingiva of moderate chronic periodontitis. Group III: inflamed gingiva of severe chronic periodontitis.

Table 2. Normalized SOD3 expressions by SOD3/ $\beta$-actin

\begin{tabular}{cccc}
\hline Sample & Group I & Group II & Group III \\
\hline 1 & 0.051 & 0.298 & 0.324 \\
2 & 0.042 & 0.189 & 0.264 \\
3 & 0.122 & 0.171 & 0.418 \\
4 & 0.084 & 0.072 & 0.472 \\
5 & 0.072 & 0.088 & 0.331 \\
6 & 0.041 & 0.093 & 0.374 \\
7 & 0.139 & 0.049 & 0.199 \\
8 & 0.078 & 0.198 & 0.399 \\
9 & 0.099 & 0.259 & 0.185 \\
10 & 0.112 & 0.211 & 0.227 \\
11 & 0.131 & 0.132 & 0.298 \\
12 & 0.216 & 0.281 & 0.322 \\
13 & 0.122 & 0.196 & 0.421 \\
14 & 0.062 & 0.262 & 0.287 \\
15 & 0.078 & 0.042 & 0.334 \\
16 & 0.082 & 0.322 & 0.449 \\
Mean \pm SD & $0.096 \pm 0.045^{\mathrm{a}}$ & $0.179 \pm 0.091^{\mathrm{b}}$ & $0.331 \pm 0.087^{\mathrm{c}}$ \\
\hline
\end{tabular}

SOD3, superoxide dismutase 3; SD, standard deviation.

${ }^{\mathrm{a}}$ Significant difference between group I and group II $(p<0.05)$. ${ }^{\mathrm{b}}$ Significant difference between group II and group III $(p<0.05)$. 'Significant difference between group I and group III $(p<0.05)$.

der of group I, group II and group III.

The quantification of SOD3 expression is shown in Table 2 and summarized as a graph in Fig. 2. The mean normalized value of SOD3 expression relative to its $\beta$-actin was $0.096 \pm 0.045$ in group I, $0.179 \pm 0.091$ in group II, 0.331 \pm 0.087 in group III. Significant difference could be found between group I and group II ( $p=0.014)$, group II and group III ( $p=0.005)$, and group I and group III $(p=0.000)(p<0.05)$.

The gingival tissue from the patients of normal group (group I), moderate periodontitis group (group II), and severe periodontitis group (group III) showed the expression of $\mathrm{HO}$ corresponding to molecular weight about $31.4 \mathrm{kDa}$ in all samples. One representative band of $\mathrm{HO}$ is shown Fig. 3. The expression levels of $\mathrm{HO}$ were increased in order of

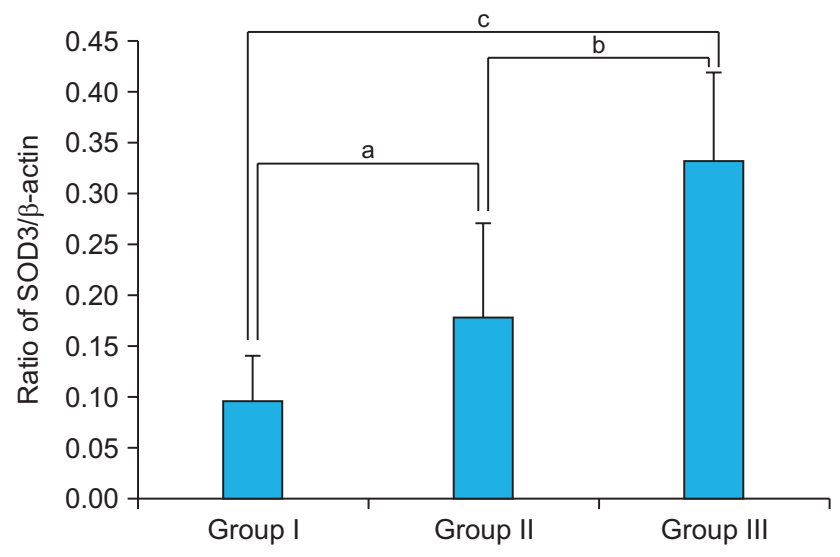

Fig. 2. Graphics showing the average amounts (ratio of superoxide dismutase 3 [SOD3]/ $\beta$-actin) and standard deviation of SOD3 level in groups I, II, and III. The levels of SOD3 in the inflamed gingiva were higher than those of healthy gingiva. ${ }^{\text {a }}$ Significant difference between group I and group II $(p<0.05)$. ' Significant difference between group II and group III $(p<0.05)$. 'Significant difference between group I and group III $(p<0.05)$.

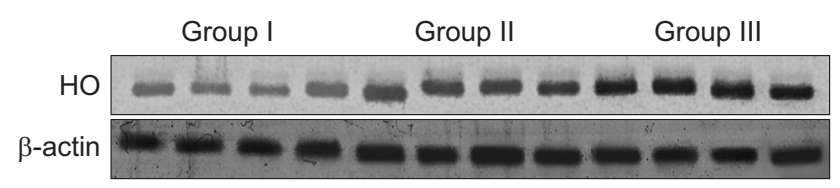

Fig. 3. Western analysis of heme-oxygenase (HO) showing 4 representative samples in each group. HO levels were quantified on the basis of $\beta$-actin levels. Group I: normal gingiva of healthy periodontal tissue. Group II: inflamed gingiva of moderate chronic periodontitis. Group III: inflamed gingiva of severe chronic periodontitis.

group I, group II and group III.

The quantification of $\mathrm{HO}$ expression is shown in Table 3 and summarized as a graph in Fig. 4. The mean normalized value of $\mathrm{HO}$ expression relative to its $\beta$-actin was $0.077 \pm$ 0.032 in group I, $0.176 \pm 0.041$ in group II, $0.299 \pm 0.057$ in group III. Significant difference could be found between group I and group II $(p=0.004)$, group II and group III $(p=0.007)$, and group I and group III $(p=0.000)(p<0.05)$.

The gingival tissue from the patients of normal group (group I), moderate periodontitis group (group II), and severe periodontitis group (group III) showed the expression of MPO corresponding to molecular weight about $84 \mathrm{kDa}$ in all samples. One representative band of MPO is shown Fig. 5. The expression levels of MPO were decreased in order of group I, group II and group III.

The quantification of MPO expression is shown in Table 
Table 3. Normalized HO expressions by $\mathrm{HO} / \beta$-actin

\begin{tabular}{cccc}
\hline Sample & Group I & Group II & Group III \\
\hline 1 & 0.034 & 0.199 & 0.334 \\
2 & 0.094 & 0.213 & 0.301 \\
3 & 0.16 & 0.211 & 0.327 \\
4 & 0.111 & 0.211 & 0.311 \\
5 & 0.044 & 0.079 & 0.299 \\
6 & 0.087 & 0.187 & 0.188 \\
7 & 0.066 & 0.157 & 0.311 \\
8 & 0.089 & 0.121 & 0.333 \\
9 & 0.052 & 0.184 & 0.333 \\
10 & 0.078 & 0.147 & 0.291 \\
11 & 0.053 & 0.236 & 0.32 \\
12 & 0.101 & 0.188 & 0.399 \\
13 & 0.071 & 0.159 & 0.288 \\
14 & 0.053 & 0.127 & 0.159 \\
15 & 0.047 & 0.183 & 0.288 \\
16 & 0.088 & 0.199 & 0.301 \\
Mean \pm SD & $0.077 \pm 0.032^{\mathrm{a}}$ & $0.176 \pm 0.041^{\mathrm{b}}$ & $0.299 \pm 0.057^{\mathrm{c}}$ \\
\hline
\end{tabular}

$\mathrm{HO}$, heme-oxygenase; SD, standard deviation.

${ }^{a}$ Significant difference between group I and group II $(p<0.05)$. ' Significant difference between group II and group III $(p<0.05)$. 'Significant difference between group I and group III $(p<0.05)$.

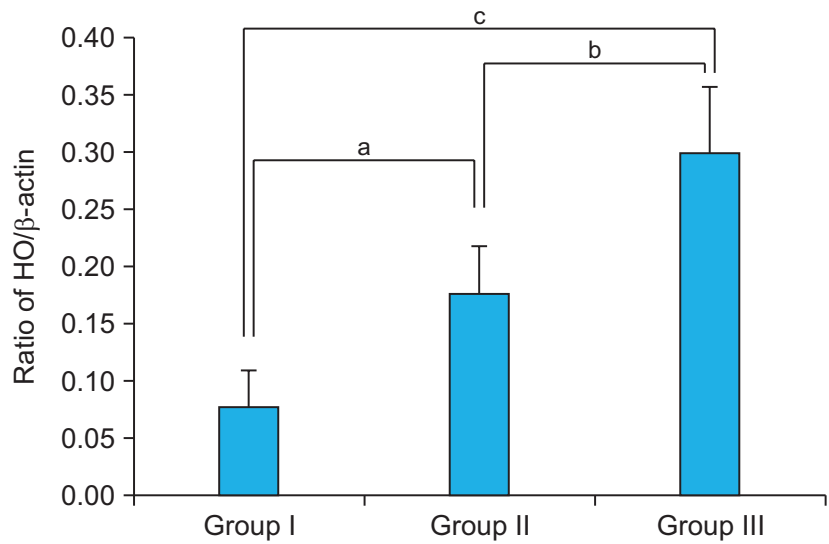

Fig. 4. Graphics showing the average amounts (ratio of IL-11/ $\beta$-actin) and standard deviation of heme-oxygenase (HO) level in groups I, II, and III. The levels of $\mathrm{HO}$ in the inflamed gingiva were higher than those of healthy gingiva. a Significant difference between group I and group II $(p<0.05)$. 'Significant difference between group II and group III $(p<0.05)$. 'Significant difference between group I and group III $(p<0.05)$.

4 and summarized as a graph in Fig. 6. The mean normalized value of MPO expression relative to its $\beta$-actin was $0.288 \pm 0.095$ in group I, $0.193 \pm 0.045$ in group II, 0.079 \pm 0.028 in group III. Significant difference could be found between group I and group II ( $p=0.011$ ), group II and group III ( $p=0.007)$, and group I and group III $(p=0.000)(p<0.05)$.

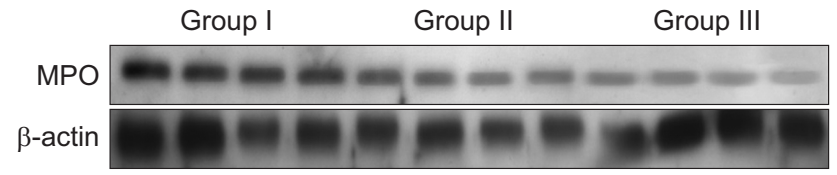

Fig. 5. Western analysis of myeloperoxidase (MPO) showing 4 representative samples in each group. MPO levels were quantified on the basis of $\beta$-actin levels. Group I: normal gingiva of healthy periodontal tissue. Group II: inflamed gingiva of moderate chronic periodontitis. Group III: inflamed gingiva of severe chronic periodontitis.

Table 4. Normalized MPO expressions by MPO/ $\beta$-actin

\begin{tabular}{cccc}
\hline Sample & Group I & Group II & Group III \\
\hline 1 & 0.112 & 0.199 & 0.088 \\
2 & 0.219 & 0.199 & 0.137 \\
3 & 0.471 & 0.247 & 0.054 \\
4 & 0.322 & 0.241 & 0.067 \\
5 & 0.324 & 0.172 & 0.051 \\
6 & 0.218 & 0.178 & 0.034 \\
7 & 0.198 & 0.099 & 0.088 \\
8 & 0.326 & 0.184 & 0.052 \\
9 & 0.424 & 0.111 & 0.137 \\
10 & 0.178 & 0.197 & 0.099 \\
11 & 0.333 & 0.211 & 0.068 \\
12 & 0.274 & 0.279 & 0.087 \\
13 & 0.388 & 0.198 & 0.087 \\
14 & 0.287 & 0.177 & 0.066 \\
15 & 0.324 & 0.174 & 0.079 \\
16 & 0.216 & 0.224 & 0.071 \\
Mean \pm SD & $0.288 \pm 0.095^{\mathrm{a}}$ & $0.193 \pm 0.045^{\mathrm{b}}$ & $0.079 \pm 0.028^{\mathrm{c}}$ \\
\hline
\end{tabular}

MPO, myeloperoxidase; SD, standard deviation.

${ }^{a}$ Significant difference between group I and group II $(p<0.05)$. ' $S i g n i f-$ icant difference between group II and group III $(p<0.05)$. 'Significant difference between group I and group III $(p<0.05)$.

\section{Discussion}

Periodontitis is the disease characterized as the host-mediated destruction of connective tissue and alveolar bone. Such destruction is induced by elevated production and activation of lytic enzymes along with stimulated osteoclastogenesis. In addition, chronic inflammation such as chronic periodontitis, is characterized by the formation of wounds and the dominant presence of macrophages, resulting in the production of reactive oxygen species resulting in tissue destruction [17].

SOD3 and $\mathrm{HO}$ are considered to be antioxidants that protect against oxidative stress. MPO is a subfamily of XPO which plays a similar role. Therefore, the purpose of this 


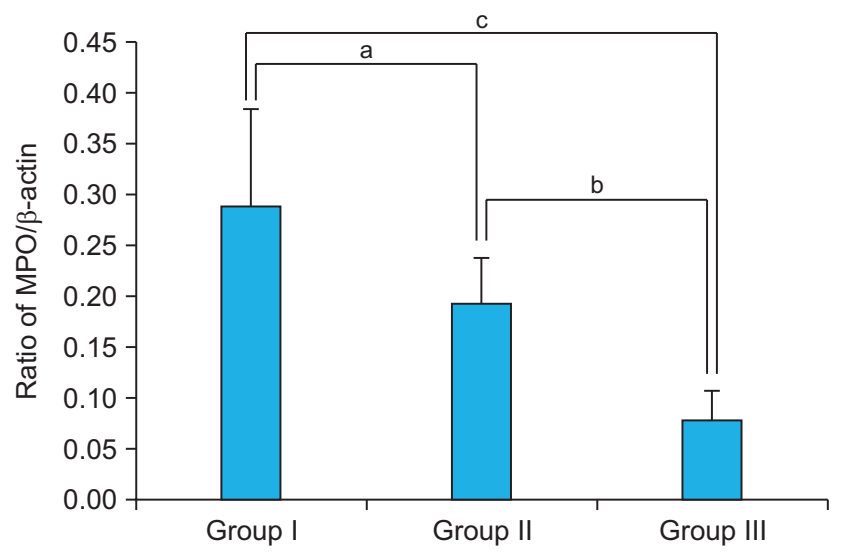

Fig. 6. Graphics showing the average amounts (ratio of MPOP/ $\beta$-actin) and standard deviation of MPO level in groups I, II and III. The levels of MPO in the inflamed gingiva were lower than those of healthy gingiva. ${ }^{a}$ Significant difference between group I and group II $(p<0.05)$. 'Significant difference between group II and group III $(p<0.05)$. 'Significant difference between group I and group III $(p<0.05)$.

study is to evaluate the effects of enzymes that play a role in preventing oxidative stress, which is closely related to chronic inflammation and tissue destruction.

Generally, SOD is considered to be a major antioxidant that plays a role in converting superoxide into relatively less harmful oxygen and hydrogen peroxide. This enzyme plays an important role in maintaining homeostasis of reactive oxygen species. There are three forms of man [18]. The role of SOD3 in the human body has not been studied much yet.

Laurila et al. [18] and Laatikainen et al. [19] suggested that SOD3 derived effects on inflammatory cytokine and adhesion molecule expression, which would explain reduced inflammation in vascular lesions, has not been properly characterized [18,19]. Moreover, Yoon et al. [20] suggested that as major cellular defensive oxidoreductases, SODs convert superoxide radicals into oxygen and hydrogen peroxide. Although there are three members of the SOD family in mammals including humans and all of three SODs share common mechanism in scavenging $\mathrm{O}_{2}^{-}$, the specific function of each isoform in periodontitis has not been studied.

In the present study, western blot was performed to evaluate the degree of SOD3 protein synthesis through samples of chronic periodontitis patients. Experimental re- sults showed that the amount of SOD3 protein synthesis increased significantly as the depth of periodontitis increased. This may be the basis for predicting the relationship between SOD3 and inflammation as previously studied.

Heme oxygenase expression is induced by oxidative stress, and in animal models increasing this expression seems to be protective. Carbon monoxide released from heme oxygenase reactions can influence vascular tone independently or influence the function of nitric oxide synthase.

Choi et al. [21] suggests that HO binds to proteins in an appropriate amount to form an essential element in various biological processes, but has a characteristic of mediating oxidative stress and inflammation when released in large quantities [21].

This process proved that $\mathrm{HO}$ was activated to alleviate inflammation and to mediate tolerance to oxidative damage. In this study, the expression of $\mathrm{HO}$ was significantly increased as the depth of periodontitis increased. This can be inferred from the results of previous studies.

The results of this study also show that the amount of protein synthesis increases as severity of periodontitis increases as SOD3 and $\mathrm{HO}$, which are expressed under oxidative stress. It is not clear yet how the two factors are synthesized through the process of inflammatory pathways, but it is expected that a clearer role will be revealed through association with the depth of inflammation.

MPO is a peroxidase enzyme that in humans is encoded by the MPO gene on chromosome 17 [22]. MPO is most abundantly expressed in neutrophil granulocytes (a subtype of white blood cells), and produces hypohalous acids to carry out their antimicrobial activity. Loria et al. [23] mentioned that MPO is found in neutrophils and monocytes, and it plays an important role in killing microorganisms.

Unlike SOD3 and HO, MPO decreased as inflammation progressed in this study, and it is known that MPO is involved in inflammation and destruction of host cells. It is conceivable that any inhibitory factor is expressed and acted to prevent the destruction process.

According to the limited results of this study, SOD3 and $\mathrm{HO}$ act as inflammatory biomarkers, while MPO is likely to play a beneficial role in regulating the inflammatory response. If these factors are combined and their charac- 
teristics can be used successfully, a more accurate diagnosis can be achieved and a more effective periodontal treatment plan can be established. Although the results of this study showed relatively clear expression patterns of SOD3 and HO as a severity of periodontitis, there are still many conflicting results, and it should be noted that only limited studies are available that directly observe the expression levels of gum tissue levels. Therefore, it is necessary to study the expression pattern of factors depending on the degree of periodontitis in gingival crevicular fluid (GCF) or gingival tissue. Based on our findings synthesized with previous ones, it is clear that $\mathrm{SOD} 3$ and $\mathrm{HO}$ are generally expressed under oxidative stress. It is also possible that these factors interact with chronic periodontitis based on a high level of correlation. Thus, these cytokines can affect osteoclast formation with different mechanisms. However, in the case of MPO, further studies on the relationship with oxidative stress are needed, but it is likely that the MPO is likely to have relevance as a member of XPO, a subfamily of peroxidase. Considering the characteristics of periodontitis that causes oxidative stress, the expression of the three factors discussed in this study should increase in terms of inducing oxidative stress. However, the actual results show that the expression of SOD3 and $\mathrm{HO}$ is increased but the expression of MPO is decreased, so there is a difference between actual and expected results. Such discrepancies may be due to the nature of cytokines whose function may vary depending on the presence of target cells, cellular environment/conditions and other cytokines. Periodontitis is an inflammatory disease associated with alveolar bone resorption and destruction of periodontal connective tissue. It is therefore important to understand the inflammatory destruction process of the soft and hard tissues and to identify the relevant factors in this process for proper diagnosis and treatment.

In conclusion SOD3 and $\mathrm{HO}$ may play a beneficial role in oxidative stress-induced periodontitis, while MPO may play a beneficial role, and these three factors may be related to the depth of inflammation seems to be. These factors are expected to be applied to diagnosis and treatment of periodontitis through more comprehensive studies.

\section{Conflicts of Interest}

The authors declare that they have no competing interests.

\section{ORCID}

\author{
Yong Yoon \\ https://orcid.org/0000-0001-9348-1410 \\ Hyerin Kim \\ https://orcid.org/0000-0001-6664-6566 \\ Jae Mok Lee \\ https://orcid.org/0000-0002-0291-6114
}

\section{References}

1. Page RC, Offenbacher S, Schroeder HE, Seymour GJ, Kornman KS. Advances in the pathogenesis of periodontitis: summary of developments, clinical implications and future directions. Periodontol 2000 1997;14:216-248. doi: 10.1111/j.1600-0757.1997.tb00199.x.

2. Graves D. Cytokines that promote periodontal tissue destruction. J Periodontol 2008;79(8 Suppl):1585-1591. doi: 10.1902/jop.2008.080183.

3. McCord JM, Fridovich I. Superoxide dismutase. An enzymic function for erythrocuprein (hemocuprein). J Biol Chem 1969;244:6049-6055. doi: 10.1016/S0021-9258(18)63504-5.

4. Raychaudhuri SS, Deng XW. The role of superoxide dismutase in combating oxidative stress in higher plants. Bot Rev 2000;66:89-98. doi: 10.1007/BF02857783.

5. D'Aiuto F, Nibali L, Parkar M, Patel K, Suvan J, Donos N. Oxidative stress, systemic inflammation, and severe periodontitis. J Dent Res 2010;89:1241-1246. doi: 10.1177/0022034510375830.

6. Kikuchi G, Yoshida T, Noguchi M. Heme oxygenase and heme degradation. Biochem Biophys Res Commun 2005;338:558-567. doi: 10.1016/j.bbrc.2005.08.020.

7. Tenhunen R, Marver HS, Schmid R. Microsomal heme oxygenase. Characterization of the enzyme. J Biol Chem 1969;244:6388-6394.

8. Ferrándiz ML, Devesa I. Inducers of heme oxygenase-1. Curr Pharm Des 2008;14:473-486. doi: 10.2174/1381612087 83597399.

9. Kinkade JM Jr, Pember SO, Barnes KC, Shapira R, Spitznagel JK, Martin LE. Differential distribution of distinct forms of myeloperoxidase in different azurophilic granule subpopulations from human neutrophils. Biochem Biophys Res Commun 1983;114:296-303. doi: 10.1016/0006$291 x(83) 91627-3$.

10. Davies MJ. Myeloperoxidase-derived oxidation: mecha- 
nisms of biological damage and its prevention. J Clin Biochem Nutr 2011;48:8-19. doi: 10.3164/jcbn.11-006FR.

11. Hampton MB, Kettle AJ, Winterbourn CC. Inside the neutrophil phagosome: oxidants, myeloperoxidase, and bacterial killing. Blood 1998;92:3007-3017. doi: 10.1182/blood. V92.9.3007.

12. Mühlemann HR, Son S. Gingival sulcus bleeding--a leading symptom in initial gingivitis. Helv Odontol Acta 1971;15: 107-113.

13. Lindhe J, Lang NP, Karring T. Clinical periodontology and implant dentistry. 5th ed. Ames: Blackwell Munksgaard; 2008.

14. Kim JB, Jung MH, Cho JY, Park JW, Suh JY, Lee JM. The influence of type 2 diabetes mellitus on the expression of inflammatory mediators and tissue inhibitor of metalloproteinases-2 in human chronic periodontitis. J Periodontal Implant Sci 2011;41:109-116. doi: 10.5051/ jpis.2011.41.3.109.

15. Jung HY, Kim YG, Park JW, Suh JY, Lee JM. The expression of a nitric oxide derivative, tissue inhibitors of metalloproteinase-3, and tissue inhibitors of metalloproteinase- 4 in chronic periodontitis with type 2 diabetes mellitus. J Periodontal Implant Sci 2013;43:87-95. doi: 10.5051/ jpis.2013.43.2.87.

16. Cho JY, Xing S, Liu X, Buckwalter TL, Hwa L, Sferra TJ, Chiu IM, Jhiang SM. Expression and activity of human $\mathrm{Na}+$ /I- symporter in human glioma cells by adenovirusmediated gene delivery. Gene Ther 2000;7:740-749. doi: 10.1038/sj.gt.3301170.

17. Bandyopadhyay U, Das D, Banerjee R. Reactive oxygen species: oxidative damage and pathogenesis. Curr Sci 1999;77:658-666.

18. Laurila JP, Laatikainen LE, Castellone MD, Laukkanen MO. SOD3 reduces inflammatory cell migration by regulating adhesion molecule and cytokine expression. PLoS One 2009;4:e5786. doi: 10.1371/journal.pone.0005786.

19. Laatikainen LE, Incoronato M, Castellone MD, Laurila JP, Santoro M, Laukkanen MO. SOD3 decreases ischemic injury derived apoptosis through phosphorylation of Erk1/2, Akt, and FoxO3a. PLoS One 2011;6:e24456. doi: 10.1371/ journal.pone.0024456.

20. Yoon Y, Kim TJ, Lee JM, Kim DY. SOD2 is upregulated in periodontitis to reduce further inflammation progression. Oral Dis 2018;24:1572-1580. doi: 10.1111/odi.12933.

21. Choi AM, Alam J. Heme oxygenase-1: function, regulation, and implication of a novel stress-inducible protein in oxidant-induced lung injury. Am J Respir Cell Mol Biol 1996;15:9-19. doi: 10.1165/ajrcmb.15.1.8679227.

22. Lau D, Baldus S. Myeloperoxidase and its contributory role in inflammatory vascular disease. Pharmacol Ther 2006;111:16-26. doi: 10.1016/j.pharmthera.2005.06.023.

23. Loria V, Dato I, Graziani F, Biasucci LM. Myeloperoxidase: a new biomarker of inflammation in ischemic heart disease and acute coronary syndromes. Mediators Inflamm 2008;2008:135625. doi: 10.1155/2008/135625. 